\title{
OTIMIZAÇÃO DO PROCESSO DE SECAGEM EM LEITO DE ESPUMA DE EXTRATO DE CAFÉ
}

\author{
Pamela Lemos Cruz¹, Sérgio Henriques Saraiva² ${ }^{2}$ Luciano José Quintão Teixeira $^{2}$ \\ 1 Mestranda no programa de Ciência e Tecnologia de Alimentos da Universidade \\ Federal do Espírito Santo (pamelaceng@gmail.com) Alegre-Brasil. \\ 2 Professor Associado do Departamento de Engenharia de Alimentos da \\ Universidade Federal do Espírito Santo.
}

Recebido em: 02/10/2017 - Aprovado em: 21/11/2017 - Publicado em: 05/12/2017 DOI: 10.18677/EnciBio_2017B45

\begin{abstract}
RESUMO
Durante o processamento do café solúvel ocorrem perdas de aromas, além de degradação e formação de compostos indesejáveis. A secagem em leito de espuma utiliza baixas temperaturas de secagem, resultando em um produto de alta qualidade, pois ocorrem menores perdas de voláteis e nutrientes durante 0 processo. O objetivo desse trabalho foi otimizar as concentrações dos agentes espumantes emustab $\AA^{\circledR}$ e soro de leite em pó no processo de secagem de extrato de café em leito de espuma, bem como avaliar o efeito da temperatura sobre a cinética de secagem. Utilizou-se um Delineamento Composto Central Rotacional (DCCR), com as concentrações dos dois agentes variando de 0 a $8 \%$. Em seguida, a secagem foi realizada em cinco temperaturas $(50,55,60,65$ e $70 \stackrel{\circ}{\circ})$ e sua cinética foi ajustada comparando-se os modelos de Page, Henderson e Pabis e Exponencial mediante uma regressão não-linear, estabelecendo a curva de secagem do processo e avaliando o efeito da temperatura sobre os parâmetros do modelo selecionado. As concentrações ótimas dos agentes espumantes foram de $0 \%$ de soro e $4 \%$ de emustab $\AA$. O modelo de Page foi o que melhor se ajustou aos dados experimentais, com um coeficiente de determinação $\left(R^{2}\right)$ superior a 0,99 em todos os casos.
\end{abstract}

PALAVRAS-CHAVE: café, café solúvel, espuma

\section{STUDY OF THE FOAM-MAT DRYING PROCESS OF COFFEE ADDED WITH DIFFERENT EMULSIFIERS}

\begin{abstract}
During the processing of soluble coffee occur losses of aromas, besides degradation and formation of undesirable compounds. Foam mat drying uses low temperatures, resulting in a high-quality product and occur less losses of volatile compounds and nutrients during the process. The objective of this work was to optimize the concentrations of emustab $\AA$ and whey powder in the foam mat drying process of coffee extract, as well as to evaluate the effect of the temperature on drying kinetics. A Central Composite Rotatable Design (CCRD) was used, with the concentrations of the two agents ranging from 0 to $8 \%$. Then, drying was performed at five temperatures $\left(50,55,60,65\right.$ and $\left.70^{\circ} \mathrm{C}\right)$ and their kinetics were adjusted by comparing the Page, Henderson and Pabis and Exponential models by a non-linear
\end{abstract}


regression, establishing the drying curves of the process and evaluating the effect of temperature on the parameters of the selected model. The optimum concentrations of the foaming agents were $0 \%$ serum and $4 \%$ emustab®. The Page model was the best fit to the experimental data, with a coefficient of determination $\left(R^{2}\right)$ over 0.99 in all cases.

KEYWORDS: coffee, foam, soluble coffee

\section{INTRODUÇÃO}

A economia cafeeira é extremamente relevante tanto no âmbito econômico quanto no social, contribuindo para a geração de empregos e fixação de mão-deobra nas áreas rurais (ABICS, 2017). As espécies mais importantes no âmbito de exportação mundial são Arabica e Canephora (OIC, 2016).

A indústria de café solúvel, em particular, tem tido grande incremento nos países tradicionalmente consumidores de café, representando em torno de $10 \%$ da colheita anual sendo direcionada para este fim (ABICS, 2017). Este fato se dá por duas grandes vantagens deste produto: Fácil e rápido preparo, reduzindo perdas que se verificam do preparo tradicional e a menor despesa de transporte, em virtude da redução de volume. Estas são duas qualidades desejáveis em um produto nos dias atuais, visto que o mundo globalizado busca a rapidez e dinamicidade inclusive no preparo de suas refeições (GMOSER et al., 2017).

Durante o processamento do café solúvel ocorrem perdas de aromas, além de degradação e formação de compostos, o que compromete a qualidade do produto final. Essas perdas ocorrem principalmente devido a ação de altas temperaturas na extração e concentração a quente e resultam no aroma e sabor característicos do café solúvel, que difere do café torrado e moído tradicional (GMOSER, et al., 2017)

Sabe-se que o café solúvel é produto resultante da desidratação do extrato aquoso de café (Coffea arábica e outras espécies do gênero Coffea) torrado e moído. Ele pode se apresentar sob a forma de pó ou grânulos. De acordo com a resolução - CNNPA no 12, de 1978, da ANVISA - o produto deve respeitar algumas diretrizes para ser comercializado em território nacional. Não são toleradas, por exemplo, quaisquer adições de conservadores ou outros aditivos (BRASIL, 1999).

A conservação por desidratação prolonga a vida útil de alimentos por permitir a remoção da água, reduzindo assim, a deterioração microbiológica e as reações de degradação, e possibilita a disponibilização de alimentos sazonais, impedindo a perda de valor comercial. Além disso, permite reduzir a massa e o volume do produto, aumentando a eficiência de transporte e de armazenamento. Também agrega valor aos alimentos, uma vez que um produto de propriedades físicas e químicas diferenciadas do mesmo in natura, é disponibilizado ao mercado (HARDY; JIDEANI, 2017).

$\mathrm{Na}$ secagem em leito de espuma, o alimento é convertido em uma espuma estável por meio da adição de agentes espumantes, como por exemplo o emustabß e soro de leite, e incorporação de ar, nitrogênio ou outros gases, em batedeiras ou outros equipamentos geradores de espuma (FELLOWS, 2006). Em seguida, a espuma é espalhada sobre uma superfície, utilizando-se geralmente camada com espessura em torno de 2 a $5 \mathrm{~mm}$. A secagem resulta em um produto poroso e quebradiço, de fácil moagem e transformação em pó com boas propriedades de reidratação (HARDY; JIDEANI, 2017).

Segundo Fellows (2006), as baixas temperaturas de secagem e os reduzidos tempos de secagem resultam em um produto de alta qualidade, pois ocorrem 
menores perdas de voláteis e nutrientes durante o processo. Além disso, a espuma aumenta a eficiência de secagem porque aumenta a área superficial e incrementa a transferência de calor e massa. Isso faz com que a secagem de uma camada de espuma seja aproximadamente três vezes mais rápida do que a secagem de uma camada similar de líquido (FELLOWS, 2006; HARDY; JIDEANI, 2017).

Dessa forma, o objetivo do presente trabalho foi estudar a secagem em leito de espuma de café adicionado de dois tipos de emulsificantes, buscando a melhor alternativa para a secagem deste produto.

\section{MATERIAL E METODOS}

O experimento foi realizado no laboratório de Operações Unitárias, localizado no Centro de Ciências Agrárias da Universidade Federal do Espírito Santo, Alegre, Espírito Santo. O café utilizado neste experimento foi o café solúvel comercial de uma marca famosa brasileira obtido no mercado local. O café foi preparado seguindo fielmente as instruções do fabricante.

Para a produção de espuma, foram utilizados dois tipos de emulsificantes, o agente espumante Emustab ${ }^{\circledR}$ que é um produto formado de mono e diglicerídeos de ácidos graxos; e soro de leite desidratado pelo método Spray Dryer doado pela Universidade Federal de Viçosa, Viçosa, Minas Gerais. Para a produção das espumas de café adicionado dos diferentes emulsificantes, foi utilizada uma batedeira doméstica na velocidade máxima durante 20 minutos nas diferentes proporções calculadas.

Para otimizar as concentrações dos agentes a serem adicionados no café para a produção de espuma e a subsequente secagem em secador convectivo, foi realizado um Delineamento Composto Central Rotacional (DCCR), com as concentrações dos dois agentes variando de 0 a $8 \%$.

Para duas variáveis fixas, como no caso, este design gera 9 tratamentos (quatro pontos fatoriais, quatro pontos axiais e um ponto central). Para um DCCR de dois fatores, é utilizado um valor de $\alpha=\sqrt{2}$ (MYERS et al., 2016). A Tabela 1 mostra os valores de concentração dos dois agentes ( $x 1$ e $x 2$ ) em cada tratamento, bem como os valores das variáveis codificadas (c1 e c2). Foram realizadas cinco repetições no ponto central, totalizando 13 experimentos.

TABELA 1. Delineamento Composto Central Rotacional Valores das variáveis fixas codificadas $\left(c_{1}\right.$ e $\left.c_{2}\right)$ e não codificadas ( $\mathrm{x}_{1}, \%$ emustab e $\mathrm{x}_{2}, \%$ soro)

\begin{tabular}{ccccc}
\hline Ensaio & $\mathbf{c}_{\mathbf{1}}$ & $\mathbf{x}_{\mathbf{1}}$ (\% de soro) & $\mathbf{c}_{\mathbf{2}}$ & $\mathbf{x}_{\mathbf{2}}$ (\% de emustab®) \\
\hline 1 & -1 & 1,17 & -1 & 1,17 \\
2 & -1 & 1,17 & 1 & 6,83 \\
3 & 1 & 6,83 & -1 & 1,17 \\
4 & 1 & 6,83 & 1 & 6,83 \\
5 & $-1,41$ & 0 & 0 & 4 \\
6 & 1,41 & 8 & 0 & 4 \\
7 & 0 & 4 & $-1,41$ & 0 \\
8 & 0 & 4 & 1,41 & 8 \\
9 & 0 & 4 & 0 & 4 \\
10 & 0 & 4 & 0 & 4 \\
11 & 0 & 4 & 0 & 4 \\
12 & 0 & 4 & 0 & 4 \\
13 & 0 & 4 & 0 & 4 \\
\hline
\end{tabular}


As condições ótimas do processo devem ser tais que minimizem as variáveis densidade da espuma, custo dos espumantes e o índice de coalescência da espuma. A Figura 1 mostra o diagrama de fluxo da produção de espuma.

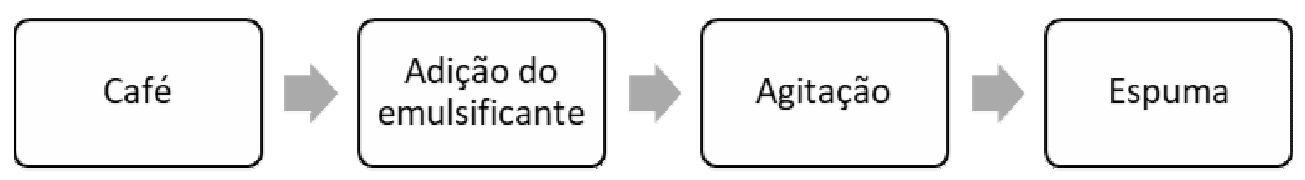

FIGURA 1 - Fluxograma da produção de espuma de extrato de café

A densidade da espuma foi determinada pela divisão da massa pelo volume através da adição da espuma em um becker de $250 \mathrm{~mL}$. Para determinação do índice de coalescência, o volume coalescido ao fundo do becker foi medido após 30 minutos e o índice é o percentual do volume coalescido em relação ao volume total da espuma.

Escolhida a melhor proporção para se obter uma espuma estável, foram preparadas espumas para os experimentos de secagem. As amostras foram espalhadas em bandejas em uma camada de $10 \mathrm{~cm}$ e seguiram para um secador com circulação de ar forçado (fluxo de ar $=0,1 \mathrm{~m} / \mathrm{s}$ ), com temperaturas controladas de $50,55,60,65$ e $70^{\circ} \mathrm{C}$ (Figura 2).

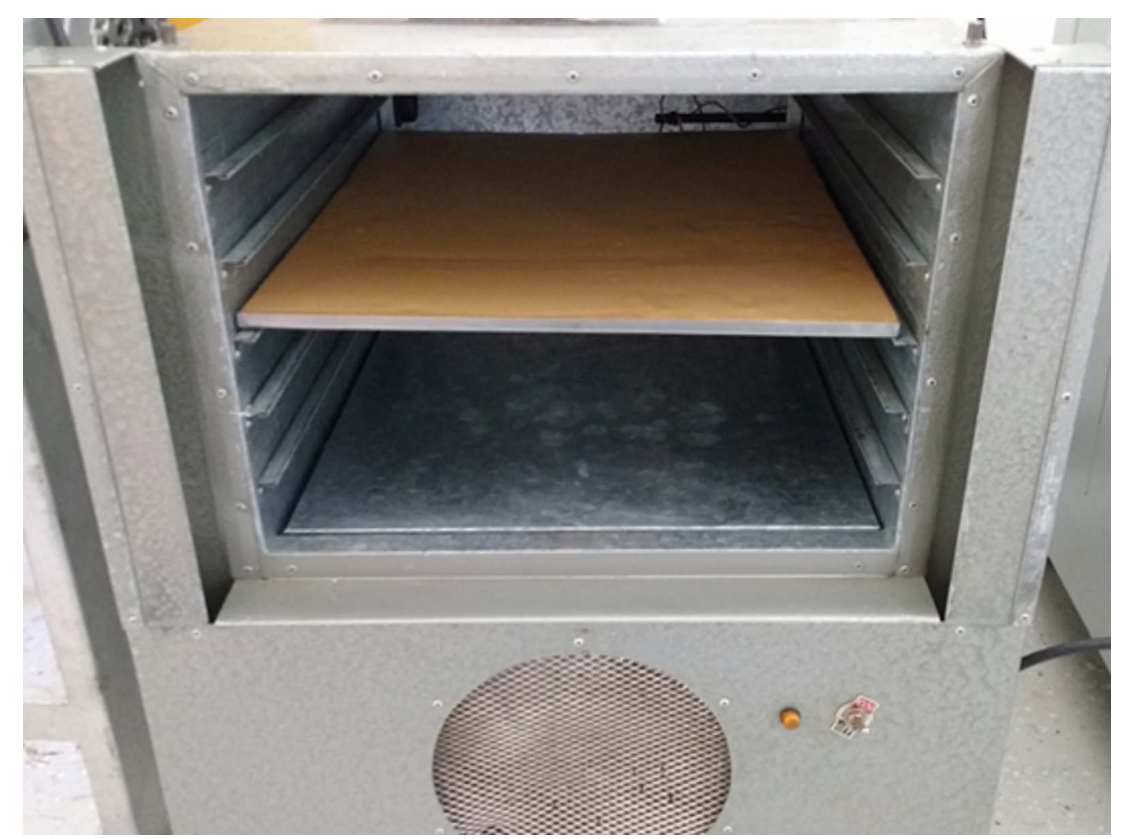

FIGURA 2 - Bandeja com espuma de extrato de café em secador convectivo

Para a determinação da cinética de secagem, o peso das amostras foi registrado a cada 15 minutos durante a primeira hora, 30 minutos durante a segunda hora e de 1 em 1 hora até peso constante.

Com os dados obtidos, foram ajustados os modelos de Page, Eq. 1, Henderson e Pabis, Eq. 2, e o exponencial, Eq.3, para cada temperatura. 


$$
\begin{aligned}
& R U=\frac{X_{t}-X_{\theta}}{X_{t}-X_{\theta}}=e^{-k t^{n}} \\
& R U=\frac{X_{t}-X_{\theta}}{X_{t}-X_{\theta}}=C e^{-k t} \\
& R U=\frac{X_{t}-X_{\theta}}{X_{t}-X_{\theta}}=e^{-k t}
\end{aligned}
$$

Em que $k, n$ e $C$ são parâmetros dos modelos, $R U$ é a razão de umidade, $X_{\mathrm{t}}$ é o teor de água em qualquer instante de tempo, $X_{e}$ é o teor de água na condição de equilíbrio, $X_{0}$ é o teor de água inicial e $t$ é o tempo de secagem em minutos.

Os modelos de curva de secagem foram ajustados por meio de análise de regressão não linear, usando a técnica de minimização da soma de quadrados dos desvios. Os modelos foram comparados com base no coeficiente de determinação $\left(R^{2}\right)$ e no erro padrão da regressão $(S)$, calculado a partir da equação seguinte:

$S=\frac{\sum_{i=1}^{n}\left(R U_{\exp i}-R U_{p r e, i}\right)^{2}}{N-p}$

Em que $R U_{\text {exp, }, i}$ e $R U_{\text {previ, }}$ são, respectivamente, os valores experimentais e previstos da razão de umidade para a i-ésima observação, $N$ é o número de observações e $p$ o número de parâmetros no modelo.

$O$ efeito da temperatura do ar de secagem sobre os parâmetros do modelo foi avaliado, segundo o modelo que melhor descreveu a cinética de secagem. Escolhido o modelo, foi construído um gráfico relacionando os parâmetros do modelo como função da temperatura. A partir da observação do comportamento de cada parâmetro do modelo, foi proposto um modelo que descrevesse a variação desse parâmetro em função da temperatura. Então, foi proposto um modelo matemático generalizado, ajustado por regressão não linear, para descrever a razão de umidade como função do tempo e da temperatura.

\section{RESULTADOS E DISCUSSÕES}

De acordo com Muthukumaran et al. (2008), a estabilidade é o atributo físico de maior importância na avaliação da qualidade da espuma. Dessa forma, é desejável que o índice de coalescência da espuma seja o menor possível para que a espuma seja estável durante todo o processo de secagem e, assim, obter um menor tempo de secagem maior facilidade de remoção do material seco das bandejas, o que resulta em um aumento da qualidade do produto final (ABBASI; AZIZPOUR, 2016). Os resultados da densidade da espuma, índice de coalescência e custo do agente espumante utilizado para os diferentes tratamentos testados são apresentados na Tabela 2. 
TABELA 2. Valores de densidade, índice de coalescência e custo para cada ensaio realizado.

\begin{tabular}{|c|c|c|c|}
\hline Ensaios & Densidade (g/mL) & $\begin{array}{c}\text { Índice de } \\
\text { coalescência (\%) }\end{array}$ & Custo $(\mathrm{R} \$ / \mathrm{g})$ \\
\hline 1 & 0,191 & 100 & 0,143 \\
\hline 2 & 0,136 & 7,94 & 0,380 \\
\hline 3 & 0,1 & 68 & 0,600 \\
\hline 4 & 0,259 & 0 & 0,833 \\
\hline 5 & 0,117 & 0 & 0,168 \\
\hline 6 & 0,242 & 6,2 & 0,808 \\
\hline 7 & 0,076 & 84 & 0,32 \\
\hline 8 & 0,184 & 0 & 0,656 \\
\hline 9 & 0,216 & 21 & 0,488 \\
\hline 10 & 0,173 & 36 & 0,488 \\
\hline 11 & 0,221 & 20,35 & 0,488 \\
\hline 12 & 0,211 & 35 & 0,488 \\
\hline 13 & 0,205 & 28 & 0,488 \\
\hline
\end{tabular}

Para as variáveis medidas experimentalmente, densidade e índice de coalescência, ajustou-se modelos de regressão linear partindo-se do modelo quadrático completo e retirando-se parâmetros não significativos. Para o custo utilizou-se um modelo de cálculo direto a partir dos custos unitários de cada agente espumante, a fim de avaliar a viabilidade do processo. A Tabela 3 apresenta os modelos ajustados para as diferentes variáveis respostas.

TABELA 3. Modelos ajustados para as diferentes variáveis respostas e forma de ajuste

\begin{tabular}{|c|c|c|c|}
\hline $\begin{array}{l}\text { Variável } \\
\text { Resposta }\end{array}$ & Equação & $\begin{array}{c}\begin{array}{c}\text { Forma } \\
\text { de } \\
\text { ajuste }\end{array} \\
\end{array}$ & $\mathbf{R}^{2}$ \\
\hline Densidade & $\rho=0,1992+0,0522 x_{1}+0,0642 x_{2}-0,0646 x_{2}^{2}+0,107 x_{1} x_{2}$ & $\begin{array}{l}\text { Regress } \\
\text { ão linear }\end{array}$ & 0,857 \\
\hline $\begin{array}{l}\text { Índice de } \\
\text { Coalescên } \\
\text { cia (\%) }\end{array}$ & $I C=28,07-14,2525 x_{1}^{2}-69,7135 x_{2}+24,6475 x_{2}^{2}$ & $\begin{array}{l}\text { Regress } \\
\text { ão linear }\end{array}$ & 0,856 \\
\hline Custo & $C=0,488+0,4527 x_{1}+0,2376 x_{2}$ & $\begin{array}{l}\text { Cálculo } \\
\text { direto }\end{array}$ & $\begin{array}{l}\text { Não se } \\
\text { aplica }\end{array}$ \\
\hline
\end{tabular}

A Tabela 4 apresenta a análise de variância da regressão e da falta de ajuste dos modelos ajustados para as variáveis: densidade da espuma e Índice de Coalescência. Observa-se que os modelos ajustados foram significativos $(p<0,05)$ e que a falta de ajuste dos modelos foi não significativa $(p>0,05)$. Esses resultados mostram que os modelos ajustados são adequados para estimar essas variáveis dentro da região experimental do trabalho.

TABELA 4. Análise de variância para os modelos ajustados por regressão linear.

\begin{tabular}{l|lllll}
\hline & F.V. & S.Q. & G.L. & Q.M. & F \\
\hline \multirow{4}{*}{ Densidade } & Modelo & 0,032512 & 4 & 0,008128 & $11,9529^{*}$ \\
& Resíduo & 0,005437 & 8 & 0,000680 & \\
& F. A. & 0,004000 & 4 & 0,001000 & $2,7840^{\text {ns }}$ \\
& E. P. & 0,001437 & 4 & 0,004368 & \\
\hline \multirow{3}{*}{ Percentual } & Modelo & 11316,27 & 3 & 3772,09 & $17,7276^{*}$ \\
coalescido & Resíduo & 1915,02 & 9 & 212,78 & \\
& F. A. & 1694,52 & 5 & 338,904 & $6,148^{\text {ns }}$ \\
& E.P. & 220,50 & 4 & 55,1240 & \\
\hline
\end{tabular}

*: Significativo $(p<0,05) ;{ }^{\text {ns: }}$ : não significativo $(p>0,05)$ 
A Figura 3 apresenta o gráfico de superfície da densidade em função das variáveis codificadas $\left(c_{1}\right.$ e $c_{2}$, vide tabela 1$)$, dentro do intervalo experimental. Notase que a densidade é menor na região próxima ao tratamento que utilizou o valor de $c_{2}$ e o valor médio de $x_{1}$, ou seja, que não utilizou emustab como agente espumante e que utilizou $4 \%$ de soro. Quanto menor a densidade da espuma, maior a incorporação de ar, ou seja, maior a capacidade de formação de espuma.

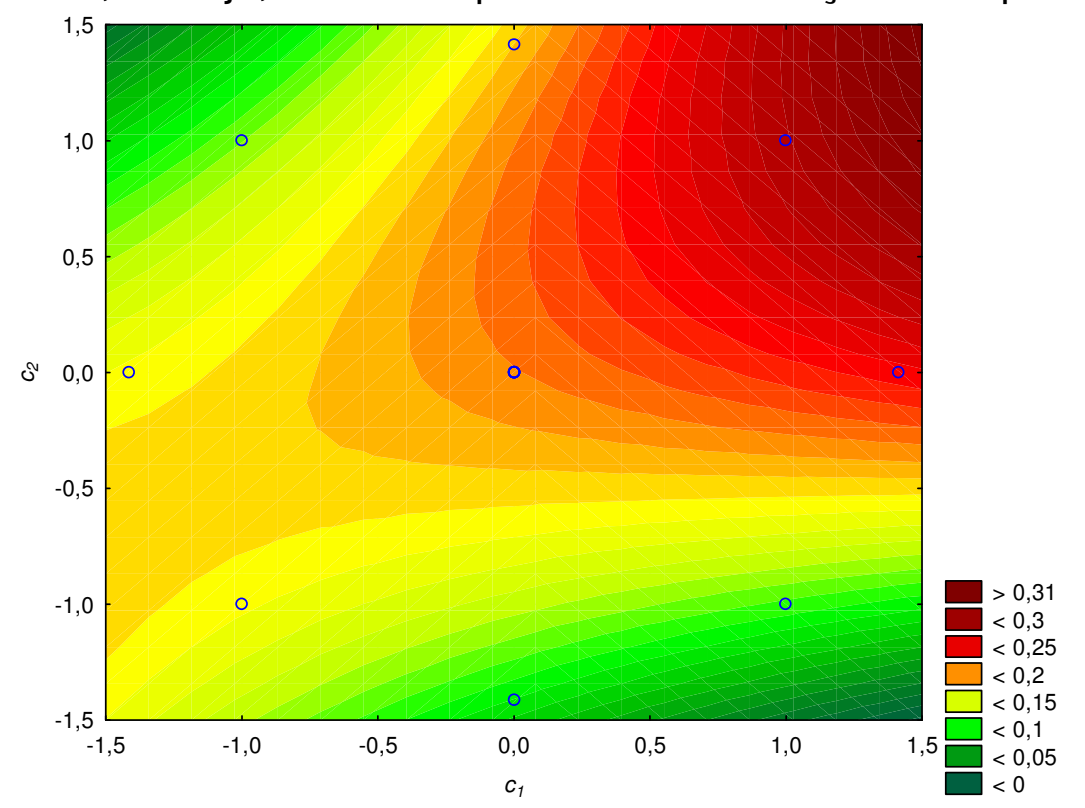

FIGURA 3. Gráfico de superfície da densidade versus as variáveis codificadas $\mathrm{C}_{1}$ e $\mathrm{C}_{2}$.

A Figura 4 apresenta o gráfico de superfície do índice de coalescência em função das variáveis codificadas $\left(c_{1}\right.$ e $c_{2}$, vide tabela 1$)$, dentro do intervalo experimental. Nota-se que o índice de coalescência é menor para a região que inclui os tratamentos com maiores valores de $x_{2}$, ou seja, maiores concentrações de emustab. O menor valor de índice de coalescência ocorreu nos tratamentos que utilizaram o valor de $x_{2}$ igual a 1 e valores de $x_{1}$ iguais a 1 e a -1 , ou seja, que utilizou $6,83 \%$ de emustab e que utilizou $1,17 \%$ ou $6,83 \%$ de soro como agente espumante. Novamente, quanto menor o índice de coalescência, maior é a estabilidade da espuma, o que é extremamente desejável para o processo de secagem em leito de espuma.

A Figura 5 apresenta o gráfico de superfície do custo do agente espumante utilizado dentro da região experimental. Nota-se que os menores custos ocorreram nos tratamentos que utilizaram os menores valores de $x_{1}$, ou seja, nas menores concentrações de soro. 


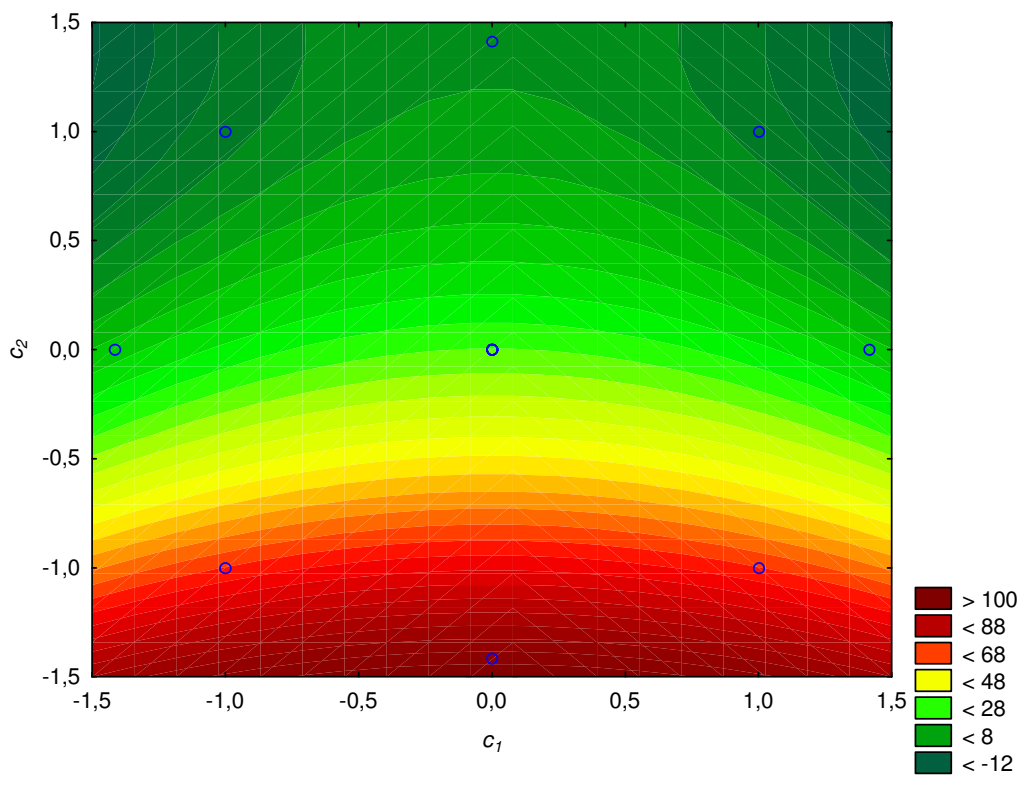

FIGURA 4. Gráfico de superfície do índice de coalescência versus as variáveis codificadas $\mathrm{c}_{1}$ e $\mathrm{c}_{2}$.

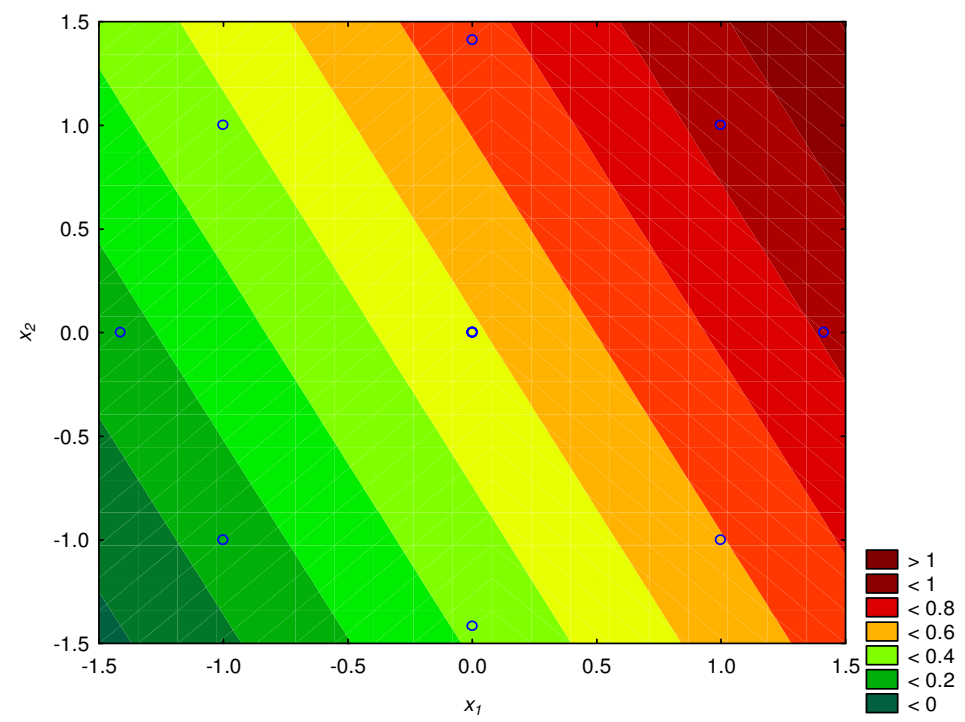

FIGURA 5. Gráfico de superfície do custo versus as variáveis codificadas $\mathrm{x}_{1}$ e $\mathrm{x}_{2}$.

As três variáveis respostas apresentam regiões ótimas distintas. As condições ótimas devem ser tais que minimizem a densidade da espuma (quanto menor a densidade, maior a incorporação de ar e, portanto, maior capacidade de formação de espuma), o percentual de coalescência (quanto menor a coalescência, maior a estabilidade da espuma) e o custo da mistura a ser usada como agente espumante.

Uma vez que as regiões ótimas para essas três variáveis são distintas, para otimizar as quantidades de soro e de emustab como agentes espumantes utilizou-se a técnica de otimização simultânea por meio da função desejabilidade (DERRINGHER; SUICH, 1980). Cada variável resposta, $y_{i}$, foi convertida em uma função desejabilidade individual, $d_{i}$, dentro do intervalo $0 \leq d_{i} \leq 1$ usando a seguinte equação: 


$$
d_{i}=\left\{\begin{array}{cc}
1 & \hat{y}_{i} \leq T_{i} \\
\left(\frac{U_{i}-y_{j}}{v_{i}-T_{i}}\right)^{r_{i}} & T_{i} \leq \hat{y}_{i} \leq U_{i} \\
0 & \hat{y}_{i}>U_{i}
\end{array}\right.
$$

Nesse trabalho utilizou-se uma função desejabilidade linear $\left(n_{1}=1 \forall i\right)$. Os valores $T_{\bar{i}}$ e $U_{\bar{i}}$ que foram considerados para cada variável resposta são apresentados na Tabela 5 . $O$ valor $\hat{y}_{i}$ para a i-ésima variável resposta foi calculado pela respectiva equação apresentada na Tabela 3.

TABELA 5 - Parâmetros da função desejabilidade para as diferentes variáveis respostas

\begin{tabular}{lll}
\hline Variável resposta & $T_{\mathrm{i}}$ & $U_{\mathrm{i}}$ \\
\hline Densidade & 0,15 & 0,25 \\
Percentual coalescido & 0,0 & 10 \\
Custo & 0,20 & 0,75 \\
\hline
\end{tabular}

A partir dos valores das desejabilidades individuais, calculou-se o valor da desejabilidade global, D, pela equação:

$$
D=\left(\Pi_{i=1}^{3} d_{i}\right)^{1 / 3}
$$

O objetivo é otimizar a desejabilidade global. A Figura 6 mostra o gráfico de superfície da função desejabilidade global como função das variáveis codificadas $x_{1}$ e $x_{2}$. Pode-se observar que a região de máxima desejabilidade $(D=1)$ inclui 0 tratamento $x_{1}$ igual a $-1,41$ e $x_{2}$ igual a 0 . Esse tratamento corresponde ao ensaio 5 (Tabela 1), o qual utiliza $4 \%$ de emustab e $0 \%$ de soro. Assim, essa proporção foi a escolhida como a condição ótima para a formação da espuma de café nas condições testadas.

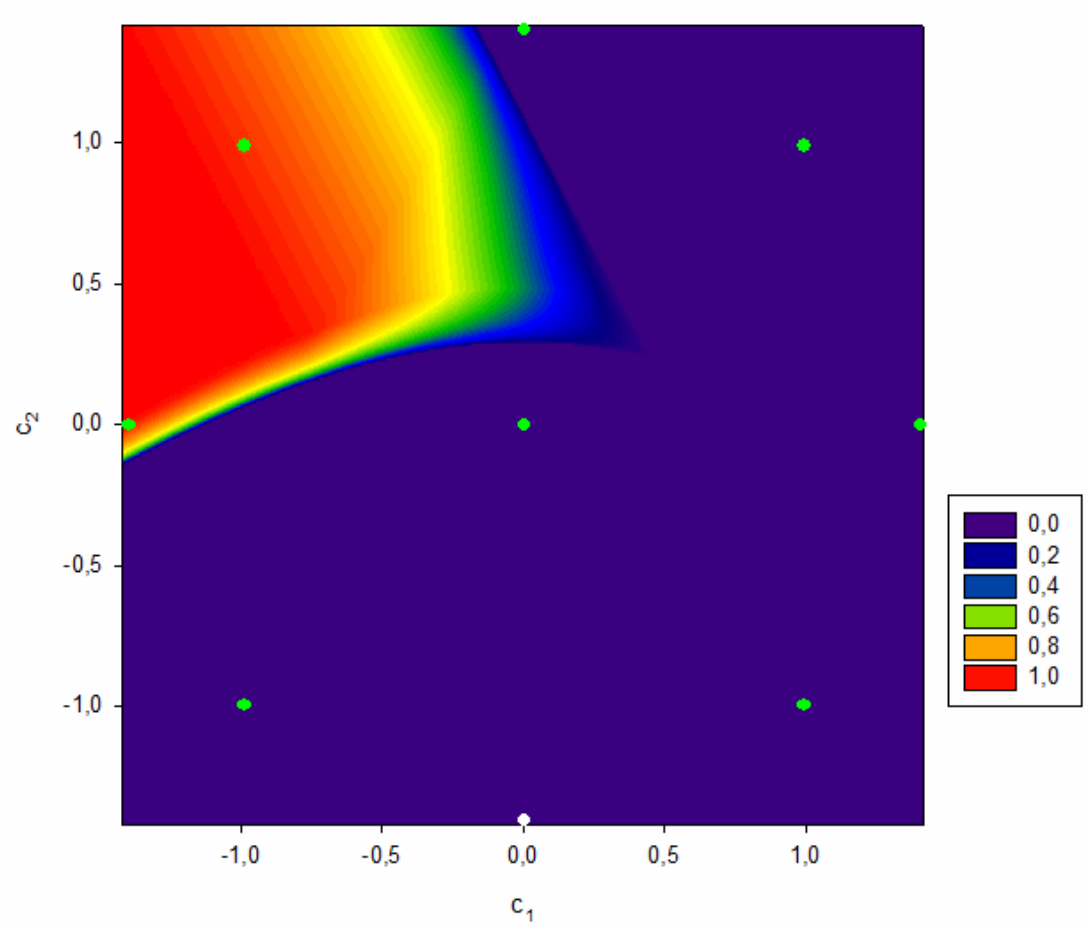

FIGURA 6. Gráfico de superfície da função desejabilidade global versus as variáveis codificadas $\mathrm{x}_{1}$ e $\mathrm{x}_{2}$. 
A partir da escolha da condição ótima de formação de espuma, a secagem foi executada. A partir dos dados experimentais de secagem, foram ajustados os modelos de Page, Henderson e Pabis (H\&P) e Lewis por meio de regressão não linear. A Tabela 6 apresenta os modelos ajustados e os seus valores de coeficientes de determinação e de erro padrão da regressão para todas as temperaturas testadas.

Todos os modelos testados foram significativos pela análise de variância da regressão e pelo teste t dos parâmetros, ambos a $5 \%$ de significância, em todas as temperaturas de secagem. Nota-se que o modelo de Page foi o que melhor se ajustou às curvas de secagem em todas as temperaturas testadas, uma vez que foi o modelo que obteve os maiores valores de coeficiente de determinação e os menores valores de erro padrão regressão. Resultados semelhantes foram obtidos por Guimarães et al. (2017) utilizando emustab para secagem de polpa de manga a 50, 60 e $70^{\circ} \mathrm{C}$. Do mesmo modo, Madureira et al. (2011) encontraram resultados semelhantes ao ajustar o modelo de Page às curvas de cinética de secagem da polpa de figo-da-índia a 50, 60 e $70^{\circ} \mathrm{C}$. Igualmente, Melo et al. (2013) encontraram a mesma conclusão ajustando este modelo a dados experimentais de cinética de secagem de espuma de fruto do mandacaru a 70,80 e $90^{\circ} \mathrm{C}$.

TABELA 6 - Modelos ajustados para curvas de secagem em diferentes temperaturas de secagem e valores de coeficientes de determinação e de erro padrão da regressão.

\begin{tabular}{|c|c|c|c|c|}
\hline Temperatura & Modelo & Equação ajustada & $\mathbf{R}^{2}$ & $s$ \\
\hline \multirow{3}{*}{$50^{\circ} \mathrm{C}$} & Page & $R U=e^{-0,006874 \mathrm{t}^{1,3413}}$ & 0,9940 & 0,0001 \\
\hline & $H \& P$ & $R U=1,0348 e^{-0,02202 t}$ & 0,9824 & 0,0023 \\
\hline & Lewis & $R U=e^{-0,02126 t} \mathrm{t}$ & 0,9807 & 0,0028 \\
\hline \multirow{3}{*}{$55^{\circ} \mathrm{C}$} & Page & $R U=e^{-0,010171 t^{1,2540}}$ & 0,9964 & 0,0001 \\
\hline & $H \& P$ & $R U=1,0322 e^{-0.02715 t}$ & 0,9886 & 0,0021 \\
\hline & Lewis & $R U=e^{-0,02631 \mathrm{t}}$ & 0,9872 & 0,0021 \\
\hline \multirow{3}{*}{$60^{\circ} \mathrm{C}$} & Page & $R U=e^{-0,005108 t^{1.5130}}$ & 0,9976 & 0,0001 \\
\hline & $H \& P$ & $R U \equiv 1,0499_{e^{-0,03864 t}}$ & 0,9769 & 0,0052 \\
\hline & Lewis & $R U=e^{-0,032287 t}$ & 0,9746 & 0,0053 \\
\hline \multirow{3}{*}{$65^{\circ} \mathrm{C}$} & Page & $R U=e^{-0,009545 t^{1.5134}}$ & 0,9978 & 0,0001 \\
\hline & $H \& P$ & $R U=1,0322 e^{-0,08821 t}$ & 0,9839 & 0,0022 \\
\hline & Lewis & $R U=e^{-0.03720 t}$ & 0,9827 & 0,0025 \\
\hline \multirow{3}{*}{$70^{\circ} \mathrm{C}$} & Page & $R U=e^{-0,01102 \mathrm{t}^{1,1435 s}}$ & 0,9974 & 0,0001 \\
\hline & $H \& P$ & $R U=1,027 e^{-0,04189 t}$ & 0,9856 & 0,0021 \\
\hline & Lewis & $R U=e^{-0.04092 \mathrm{t}}$ & 0,9848 & 0,0022 \\
\hline
\end{tabular}

As curvas de secagem ajustadas e experimentais para as diferentes temperaturas de secagem testadas são mostradas na Figura 7. Observa-se que o modelo de Page foi o que mais se aproximou dos dados experimentais em todas as temperaturas testadas, confirmando que, dentre os três modelos utilizados, ele foi o melhor. 

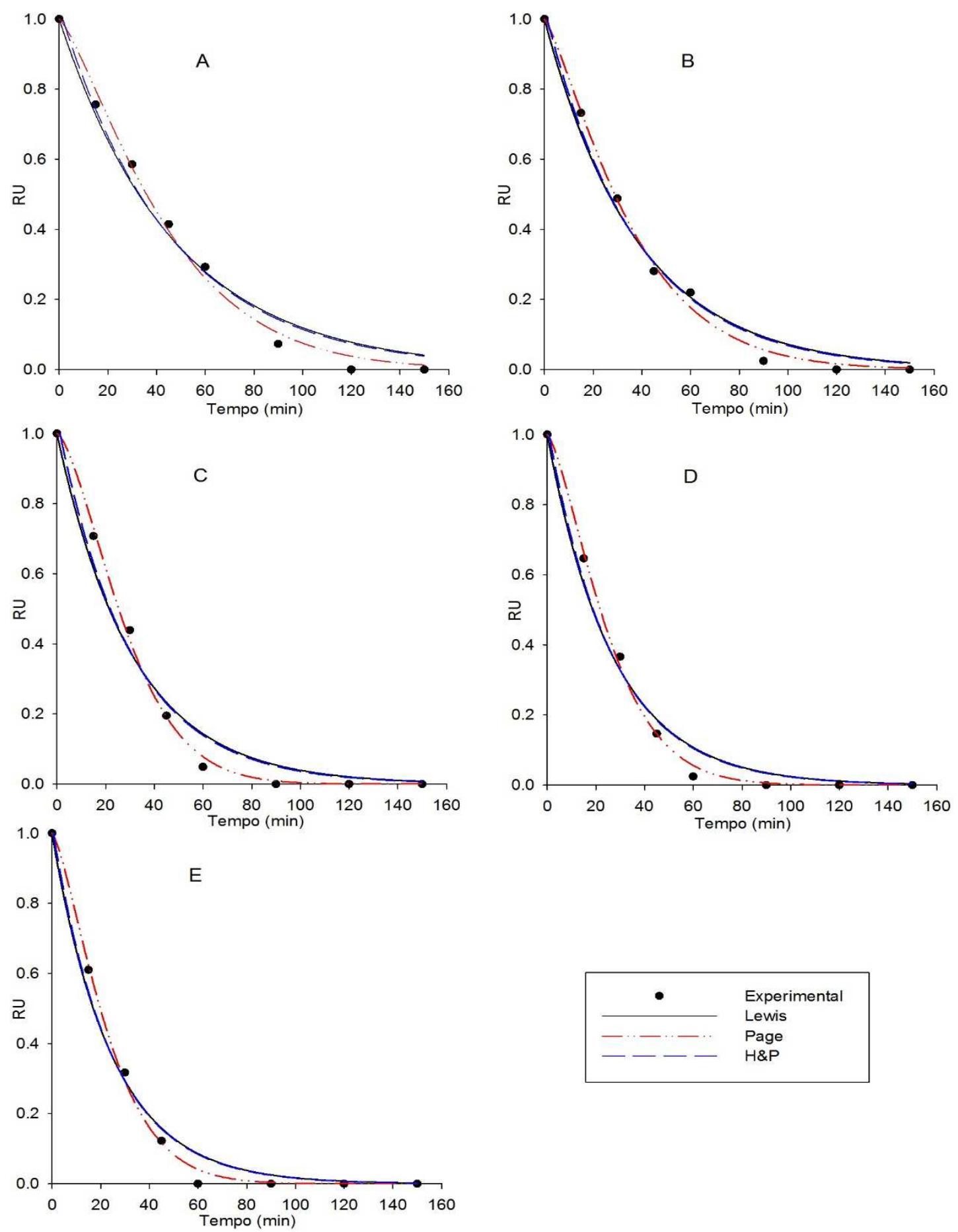

FIGURA 7 - Curvas de secagem experimentais e ajustadas pelos modelos de Page, $\mathrm{H} \& \mathrm{P}$ e Lewis para as seguintes temperaturas de secagem: A: $50^{\circ} \mathrm{C}$; $\mathrm{B}$ : $55^{\circ} \mathrm{C}$; C: $60^{\circ} \mathrm{C}$; D: $65^{\circ} \mathrm{C}$ e $\mathrm{E}: 70^{\circ} \mathrm{C}$.

Escolhido o modelo de Page como o que melhor descreve a cinética de secagem da espuma em estudo, foi feita uma avaliação sobre como a temperatura influencia nos parâmetros do modelo de Page. Inicialmente calculou-se o coeficiente de variação desses parâmetros obtidos nas diferentes temperaturas. Para 0 parâmetro $k$ o coeficiente de variação foi de $28,8 \%$ e para o parâmetro $n$ o coeficiente de variação foi de $7,6 \%$. Esses resultados indicam que o parâmetro $n$ variou muito pouco dentro do intervalo de temperatura testado e que o parâmetro $k$ 
variou de forma mais pronunciada dentro desse intervalo. Assim, para a obtenção de um modelo generalizado, em que a razão de umidade seja função da temperatura e do tempo de secagem, escolheu-se um modelo com a forma do modelo de Page, no qual o parâmetro $k$ seja uma função da temperatura e o parâmetro $n$ seja independente da temperatura, ou seja,

e

$$
k=f(T)
$$

$$
R U=e^{-[f(T)] t^{n}}
$$

Uma vez que o parâmetro $k$ está relacionado com a velocidade de secagem, ele depende diretamente da difusividade efetiva do vapor d'água no processo de secagem. Um dos modelos mais usados para descrever a variação da difusividade efetiva em função da temperatura de secagem é o modelo de Arrhenius. Assim, escolheu-se como $f(T)$ uma função com o mesmo formato que a Equação de Arrhenius, conforme Eq.9.

$$
k=f(T)=k_{1} e^{\frac{-k_{2}}{T}}
$$

Substituindo a Eq.9 na Eq.8 obtém-se o modelo generalizado:

$$
R U=e^{-\left(k_{1} e^{\frac{-k_{2}}{T}}\right) t^{n}}
$$

Em que a razão de umidade, $R U$, é função da temperatura $T$, em kelvin, e do tempo $t$, em min e $k_{1}, k_{2}$ e n são parâmetros do modelo.

Utilizando todos os dados experimentais das curvas de secagem de todas as temperaturas testadas, os parâmetros da Eq.10 foram ajustados por meio de regressão não linear, obtendo-se o seguinte modelo:

$$
R U=e^{-\left(17659,94 e^{\frac{-4757,82}{T}}\right) t^{1,3553}}
$$

Esse modelo foi significativo $(p<0,05)$ pela análise de variância da regressão e pelo teste t para os parâmetros, tendo obtido um coeficiente de determinação de 0,9950 . A Figura 8 mostra o gráfico dos valores preditos pelo modelo generalizado versus os valores experimentais. Nota-se que os valores estão próximos e aleatoriamente dispersos em torno da bissetriz (função identidade), o que indica uma boa qualidade do ajuste do modelo generalizado. Na prática, este modelo é de suma importância para determinar os principais parâmetros do processo de secagem (razão de umidade, temperatura e tempo) de forma a otimizar o processo conforme a necessidade do produtor. 


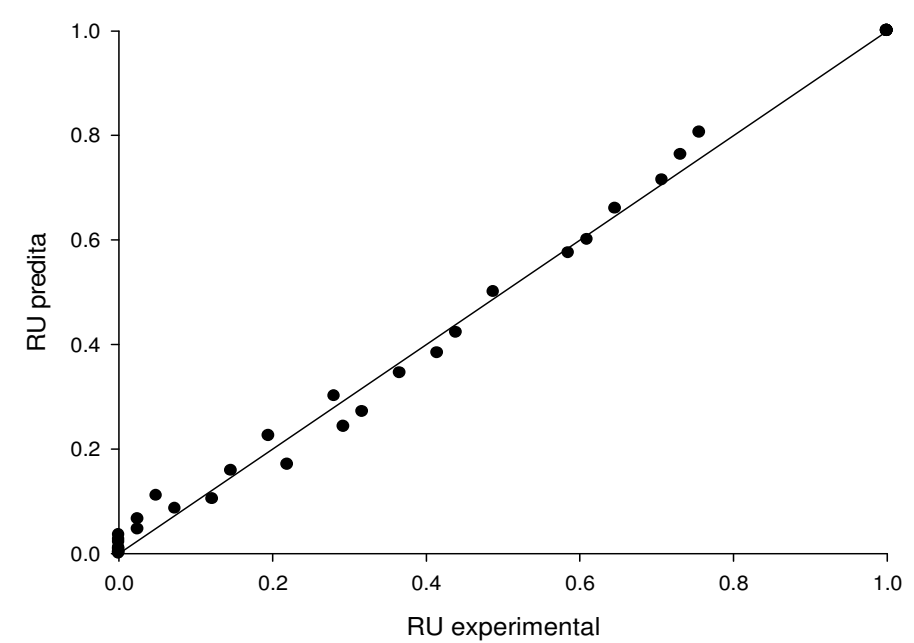

FIGURA 8 - Valores de razão de umidade preditos pelo modelo generalizado versus os valores de razão de umidade experimentais. A linha cheia representa a função identidade.

\section{CONCLUSÕES}

Nas condições estabelecidas e válidas para o estudo pode-se inferir que a melhor proporção de emulsificante testada para a formação de uma espuma estável para secagem de extrato de café em leito de espuma foi a de $4 \%$ de emustab e $0 \%$ de soro de leite, considerando a otimização das variáveis de suma importância para a viabilidade do processo.

O modelo de Page foi o que melhor se adequou às curvas de secagem dentre os modelos testados, apresentando maiores valores de coeficiente de determinação $\left(R^{2}\right)$ e menores valores de erro padrão da regressão em todas as temperaturas.

Foi observado que 0 parâmetro $k$ do modelo de Page apresentou variabilidade das diferentes temperaturas de processo, aumentando seu valor com a elevação da temperatura do ar de secagem, podendo assim ter seus valores ajustados pelo modelo de Arrhenius. Com isso a combinação do modelo de Page juntamente com o de Arrhenius gerou um novo modelo generalizado da razão de umidade em função do tempo e temperatura, podendo este ser utilizado para prever os parâmetros do processo de secagem conforme a necessidade do produtor.

Dessa forma, o processo de secagem em leito de espuma de café pode ser aplicado de maneira viável e otimizada de acordo com os parâmetros definidos neste estudo.

\section{AGRADECIMENTO}

Os autores agradecem ao Conselho Nacional de Desenvolvimento Científico e Tecnológico (CNPq) pelo financiamento deste trabalho, sem este auxílio, não seria possível a realização do mesmo.

\section{REFERÊNCIAS}

ABBASI, E., AZIZPOUR, M., Evaluation of Physicochemical Properties of Foam Mat Dried Sour Cherry Powder, LWT - Food Science and Technology, 2016. Disponível em: <https://doi.org/10.1016/j.Iwt.2015.12.004>. doi: 10.1016/j.Iwt.2015.12.004

ABIC - Associação Brasileira da Indústria Do Café. Produção agrícola, 2015. ENCICLOPÉDIA BIOSFERA, Centro Científico Conhecer - Goiânia, v.14 n.26; p.490 
Disponível

em:

<http://www.abic.com.br/publique/cgi/cgilua.exe/sys/start.htm?sid=48>. Acesso em: 18 agosto. 2017

ABICS - Associação Brasileira da Indústria do Café Solúvel. Notícias, 2017. Disponível em: <http://www.abics.com.br/secao/informacoes/noticias/>. Acesso em: 10 agosto. 2017

ABICS - Associação Brasileira da Indústria do Café Solúvel. Fabricação, 2017. Disponível em: < http://www.abics.com.br/fabricacao/>. Acesso em: 05 agosto de 2017

BRASIL. Agência Nacional de Vigilância Sanitária (ANVISA). Portaria n 130, de 19 de fevereiro 1999. Aprova o Regulamento Técnico referente a café solúvel. Diário oficial da República Federativa do Brasil, Brasília, DF, 19 fev. 1999. Disponível em: <http://www.anvisa.gov.br/anvisalegis/portarias/130_99.htm>. Acesso em: 27 de agosto de 2017

DERRINGER, G.; SUICH, R. Simultaneous Optimization of Several Response Variables. Journal of Quality Technology, v. 12, n. 4, p. 214-219, 1980. QICID: 5341. Disponível em: <http://asq.org/qic/display-item/?item=5341>. Acesso em: 18 setembro de 2017

FELLOWS, P. J. Tecnologia do processamento de alimentos: Princípios e prática. 2 ed. Porto Alegre: Artmed, 2006. 602p

GMOSER, R.; BORDES, R.; NILSSON, G.; ALTSKÄR, A.; STADING, M.; LORÉN, N.; BERTA, M. Effect of dispersed particles on instant coffee foam stability and rheological properties. European Food Research and Technology, v. 243, n. 1, p. 115-121, 2017. Disponível em: <https://doi.org/10.1007/s00217-016-2728-7>. doi: $10.1007 / \mathrm{s} 00217-016-2728-7$

GUIMARÃES, M.K.A; FIGUEIRÊDO, R.M.F.; QUEIROZ, A.J.M. Foam-mat drying kinetics of keitt mango pulp. Revista Caatinga, v. 30, n. 1, 2017. Disponível em <http://dx.doi.org/10.1590/1983-21252017v30n119rc>. doi: 10.1590/198321252017v30n119rc

HARDY, Z.; JIDEANI, V.A. Foam-mat drying technology: A review. Critical Reviews in Food Science and Nutrition, v. 57, n. 12, 2017. <http://dx.doi.org/10.1080/10408398.2015.1020359>. doi: 10.1080/10408398.2015.1020359

MADUREIRA, I. A. M. et al. Cinética de secagem da polpa do figo-da-índia. Revista Brasileira de Produtos Agroindustriais , Campina Grande, v. 13, Sup., p. 345-354, 2011. Disponível em <http://www.deag.ufcg.edu.br/rbpa/rev13e/Art13E3.pdf>. ISSN 1517-8595

MELO, K. S. et al. Secagem em camada de espuma da polpa do fruto do mandacaru: experimentação e ajustes de modelos matemáticos. Revista Caatinga, 
Mossoró, v. 26, n. 2, p. 10-17, 2013. Disponível em: $<$ https://periodicos.ufersa.edu.br/index.php/sistema>. ISSN 1983-2125 (online)

MUTHUKUMARAN, A.; RATTI, C.; RAGHAVAN, V. G. S. Foam-mat freeze drying of egg white-mathematical modeling part II: freeze drying and modeling. Drying Technology, v. 26, p. 513-518, 2008. Disponível em: <http://dx.doi.org/10.1080/07373930801929615>. doi: 10.1080/07373930801929615

MYERS, R.H., MONTGOMERY, D.C., ANDERSON-COOK, C.M. Response surface methodology: Process and product optimization using designed experiments. Hoboken, N.J.: Wiley, 2016. 855p

OIC - Organização Internacional do café. Estatísticas do comércio. 2016. Disponível em:

http://www.ico.org/pt/trade_statisticsp.asp?section=Estat\%EDstica>. Acesso em: 15 agosto. 2017 